\title{
Detection of the Ether Using the Global Positioning System
}

\author{
Stephan J. G. Gift ${ }^{1}$ \\ ${ }^{1}$ The University of the West Indies, St. Augustine, Trinidad and Tobago, West Indies \\ Correspondence: Stephan J. G. Gift, Department of Electrical and Computer Engineering, Faculty of Engineering, The \\ University of the West Indies, St. Augustine, Trinidad and Tobago, West Indies. E-mail: Stephan.Gift@sta.uwi.edu
}

Received: September 20, 2020

Accepted: October 10, 2020

Online Published: April 7, 2021

doi:10.5539/apr.v13n1p12

URL: http://dx.doi.org/10.5539/apr.v13n1p12

"If future experiments were to reveal a non-zero aether drift, then Einstein's relativity would crumble."

Diana Buchwald and Kip Thorne, 2004

\begin{abstract}
In this paper the latest evidence for the existence of a cosmic ether obtained using modern technology is reviewed. The synchronized clocks of the GPS are applied in the search for ether drift by direct measurement of light travel times in the East-West direction. This method reveals that light travels faster West than East and therefore indicates the existence of an Earth-bound ether which we identify as the Earth-centered Inertial (ECI) frame for light transmission. The GPS clocks are then applied in the search for ether drift by direct measurement of light travel times in a modified Michelson-Morley experiment. The East-West light speed difference enables the unambiguous detection of ether drift and the direct confirmation of the existence of a preferred frame. The range equation of the GPS that operates in the Earth-centered inertial (ECI) frame is employed to demonstrate ether drift for rotational motion and Time Transfer technology involving a geo-stationary GPS satellite provides further confirmation of ether drift resulting from the rotating Earth. Finally, using a model applicable in the sun-centered inertial (SCI) frame with Coordinated Universal Time, light speed variation arising from the Earth's orbital motion for light reflected from planets and spacecraft and received at the surface of the Earth is demonstrated. The evidence then is that modern technology has detected ether drift for rotational and orbital motion from the frame of the moving Earth.
\end{abstract}

Keywords: Global Positioning System, Ether, ECI Frame, Range Equation, Time Transfer SCI Frame

\section{Introduction}

At the turn of the 19th century there was considerable evidence supporting the existence of the hypothesized ether, a medium permeating all space through which electromagnetic waves were believed to travel (Whittaker, 1973; Schaffner, 1972). This medium was argued by Maxwell to support the propagation of electromagnetic waves at a speed $c=1 / \sqrt{\varepsilon_{o} \mu_{o}}$ where the electric permittivity $\varepsilon_{o}$ and the magnetic permeability $\mu_{o}$ are defined and measurable characteristics of that medium. The value of light speed calculated using measured values of $\mathcal{E}_{o}$ and $\mu_{o}$ was experimentally confirmed by timing light transmission over a specified distance. This striking confirmation strengthened belief in the existence of the ether as the medium of light travel. In search of further direct evidence of its existence, the famous Michelson-Morley experiment of 1887 (Michelson \& Morley, 1887) was conducted in an attempt to detect movement of the Earth through this medium. This experiment involved interfering light beams that traversed orthogonal paths on a movable apparatus. It was designed to reveal the speed of the Earth's orbital motion through the hypothesized ether based on the expected changes in light speed arising from movement with or against the associated ether wind. The recorded fringe shift was significantly less than the value expected as a result of the orbiting Earth and the essentially null result was interpreted as an indication of light speed constancy in all directions on the surface of the Earth. This idea of light speed invariance later formed the basis of the special theory of 
relativity.

In a recent paper DeMeo (DeMeo, 2014) reviewed some of the empirical data associated with this experiment and the search for a cosmic ether. He focused specifically on the extensive Michelson-Morley type experiments of Dayton Miller (Miller, 1933) which seemed to suggest ether-drift detection in a specific direction through the cosmos. He also discussed the rebutting paper by Shankland et al. (Shankland, Cuskey, Leone, \& Kuerti, 1955) that attributed the fringe shifts observed by Miller to temperature variations as a result of which Dayton Miller's results have been largely forgotten. Modern versions of these experiments use electromagnetic resonators in search of light speed anisotropy (Brillet \& Hall, 1979; Hermann, Senger, Kovalchuk, Muller, \& Peters, 2005; Müller, Stanwix, Tobar, Ivanov, Wolf, Herrmann, Senger, Kovalchuk, \& Peters, 2007; Eisele, Nevsky, \& Schiller, 2009). They compare the resonant frequencies of two orthogonal resonators and seek to detect light speed changes arising from orbital or rotational motion of the Earth. These experiments conducted in the frame of the rotating Earth have progressively lowered the limit on light speed anisotropy to a value of $\delta c / c<10^{-17}$ where $\delta c$ is the measured change in light speed.

Now it is well known that a major weakness of Michelson-Morley type experiments (in vacuum) which search for ether drift is they are second-order i.e. the effect to be observed is proportional to $\left(v^{2} / c^{2}\right)$ where $v$ is the speed of the Earth through the ether, since the light travels out-and-back resulting in the cancellation of first-order effects. Such experiments must therefore be quite sensitive since second-order effects are significantly smaller than first-order effects. Moreover, second-order experiments are directly affected by the second-order effects of time dilation (observed to be true) and length contraction (inferred to be true) thereby rendering these experiments unreliable for ether drift detection. In fact, Gift (Gift, 2010a) and Levy (Levy, 2003) have shown that in ether theory, two-way light speed measurement will always give the light speed in the underlying light-carrying ether medium. This finding is independent of the movement of the platform on which the measurement is being made. This amazing result means that two-way light speed measurement will always give a constant value as has been experimentally confirmed (Zhang, 1997), thereby giving the illusion of one-way light speed invariance. It follows therefore that second-order experiments are unsuitable for ether drift detection; This should be done using first-order tests.

Following the failure of the Michelson-Morley experiment in 1887 to detect the orbital motion of the Earth through the ether, Michelson in 1904 (Michelson \& Gale, 1904) did indeed suggest a first-order test to detect the rotational motion of the Earth through the ether. He proposed that if the ether is not entrained by the rotating Earth then two rays of light circumnavigating the Earth in opposite directions would do so in measurably different times that are dependent on the rotational speed $w$ of the Earth. This is because the ray travelling westward would do so at a speed $c+w$ while the ray travelling eastward would do so at a speed $c-w$. In 1925 Michelson and Gale (Michelson \& Gale, 1925) tested and exactly confirmed the predicted fringe shift arising from the rotating Earth. They showed that it was possible to detect the rotation of the Earth through a fixed ether by measuring its effects on the speed of light using a very large rectangular path fixed on the surface of the Earth. This first-order experiment was successfully repeated with greater precision by Bilger et al. (Bilger et al., 1995) using laser technology and on a global scale in 1985 by Allen et al using orbiting satellites (Allan, Weiss, \& Ashby, 1985). Earlier in 1913, Sagnac conducted a table-top version of this experiment and was the first to demonstrate fringe shifts arising from movement through the ether (Sagnac, 1913).

In the spirit of the search for ether drift in the failed Michelson and Morley experiment, the Sagnac experiment and 
later the Michelson-Gale experiment positively detected ether drift arising from rotation, confirming the existence of an ether close to the surface of the Earth. This Earth-local or Earth-bound ether is today referred to as the Earth-Centered Inertial (ECI) frame. It is a frame that moves with the Earth but does not share its rotation, just as Michelson theorized. In this frame light travels at constant speed $c$ as set by the permeability $\mu_{o}$ and permittivity $\mathcal{E}_{o}$ of the medium, just as Maxwell argued. The detection of this Earth-bound ether by the Sagnac and Michelson-Gale experiments with the expected characteristics is an incontrovertible fact, even though it is not acknowledged by mainstream science. Moreover, the existence of this Earth-bound ether or ECI frame which is integral to the operation of the GPS, directly refutes special relativity that prohibits preferred frames! As Buchwald and Thorne stated, "If future experiments were to reveal a non-zero aether drift, then Einstein's relativity would crumble" (Buchwald \& Thorne, 2005).

While the Michelson-Gale experiments gave results consistent with the differences in light travel time predicted by ether drift for light travelling in opposite directions around the rotating Earth, like the Michelson-Morley experiments they do not directly measure these time differences and therefore only indirectly indicate light speed variation. This has allowed claims that the observed fringe shift is also explainable by special relativity (Post, 1967) and hence is not necessarily an indication of an ether which special relativity rejects. That argument is today negated by the established existence of the ECI frame which is an Earth-bound ether that contradicts special relativity, independently of any explanations of the fringe shifts by the theory!

It is possible to rebut this line of argument supporting special relativity in another way. Since as previously noted special relativity demands constant light speed on the rotating Earth (Rindler, 2006) and therefore no east-west time differences as arising from ether drift, what was needed was a direct east-west measurement of one-way light transmission time between two points fixed on the surface of the rotating Earth such as was attempted by Allen et al. (Allen et al., 1992). In this regard, Bethell quoted from an 1875 article in the Encyclopedia Britannica in which Maxwell stated (Bethell, 2009), "If it were possible to determine the velocity of light by observing the time it takes [for light] to travel between one station and another on the earth's surface, we might by comparing the observed velocities in opposite directions, determine the velocity of the ether with respect to these terrestrial stations."

The Global Positioning System (GPS) contains the technology needed to implement this idea and thereby provides a new and interesting approach to ether drift detection. It is a modern navigational system that employs accurate synchronized atomic clocks in its operation (Xu, 2007). These clocks enable the accurate determination of time and are employed in a range of real-world applications including time-stamping of commercial transactions, network synchronization and the direct measurement of light travel times. According to the IS-GPS-200E Interface Specification (IS-GPS-200E, 2010), GPS signals propagate in straight lines at the constant speed $c$ (in vacuum) in the Earth-Centered Inertial (ECI) frame. This constancy of the speed of light in the ECI frame has been exhaustively confirmed and is utilized in the range equation of the GPS to accurately determine the instantaneous position of objects which are stationary or moving on or near the surface of the Earth. Light speed constancy in the ECI frame also enables the accurate synchronization of clocks which are stationary in or moving through this frame such as clocks fixed on the surface of the rotating Earth (Ashby, 2003).

Therefore, instead of using interferometry in a second-order experiment to indirectly deduce time differences in light travel arising from ether drift, such time differences can now be directly measured using synchronized clocks of the GPS. Marmet (Marmet, 2000) using data from the GPS observed that light takes longer traveling eastward from San Francisco to New York as compared with the signal traveling westward from New York to San Francisco. Kelly (Kelly, 2005) also noted that measurements using the GPS reveal that a light signal takes some 414 nanoseconds longer to circumnavigate the Earth eastward at the equator than a light signal travelling westward around the same path. These two researchers concluded that these observed travel time differences in the synchronized clock measurements in each direction occur because light travels at speed $c-v$ eastward and $c+v$ westward relative to the surface of the earth consistent with ether drift arising from the rotating Earth. Here $v$ is the speed of rotation of the Earth's surface at the particular latitude. This important research was followed by a series of papers by this author describing the use of GPS technology in confirming one-way light speed $c-v$ eastward and $c+v$ westward relative to the surface of the Earth. These results not only re-confirm the existence of the Earth-bound ether or ECI 
frame, but completely invalidate special relativity which requires constant light speed in all directions.

Regarding detection of ether drift resulting from orbital motion of the Earth, while the previous techniques enabled detection of ether drift arising from the rotation of the Earth within the ECI frame, as previously indicated the 1887 Michelson-Morley experiment tried unsuccessfully to detect the orbital motion of the Earth and this ultimately resulted in the abandonment of the ether as the all-pervasive carrier of electromagnetic radiation. In a paper in Nature marking the centenary of the introduction of relativity theory, John Stachel stated (Stachel, 2005) "all attempts to detect the translational motion of the Earth through the ether by means of optical, electrical or magnetic effects consistently failed. Lorentz succeeded in explaining why: according to his theory, no such effect should be detectable by any experiment sensitive to first order in $(v / c)$ where $v$ is the speed of the moving object through the ether and $c$ is the speed of light in that medium." This statement about Lorentz's theory is quite misleading! What this theory in fact established is that in experiments utilizing closed light paths, no first-order effects are detectable since in such experiments first-order terms cancel (Mansouri \& Sexl, 1977). Lorentz himself stated (Lorentz, 1905), "The problem of determining the influence exerted on electric and optical phenomena by a translation, such as all systems have in virtue of the Earth's annual motion, admits of a comparatively simple solution, so long as only those terms need be taken into account, which are proportional to the first power of the ratio between the velocity of translation $v$ and the velocity of light $c$. Cases in which quantities of the second order, i.e. of the order $v^{2} / c^{2}$, may be perceptible, present more difficulties." He cited the Michelson-Morley experiment as one example of such a second-order experiment that was ultimately unsuccessful.

Thus first-order open-path experiments seeking to detect the translational motion of the Earth are superior to second-order experiments since as already indicated, first-order effects are larger and therefore more easily detected than second-order effects. Additionally, unlike second-order experiments, first-order experiments are unaffected by the second-order effects of length contraction and time dilation. However, in the past, technical difficulties prevented the execution of any open-path first-order experiments. For example, Maxwell suggested a first-order approach to detecting the galactic movement of the Earth using the delay in the eclipse time of Jupiter's satellite Io first observed by Roemer (Saito, 2005). Unfortunately, inadequate equipment sensitivity did not allow the test to be conducted at that time.

Since then, in a test similar to Maxwell, Gift (Gift, 2006) used the Doppler effect for light from a star on ecliptic and the Roemer effect for light from Jupiter's satellite Io to successfully detect the orbital motion of the Earth through the ether. This Sun-local or solar-bound ether is the Sun-Centered Inertial (SCI) frame through which it is known that light travels at constant speed c. It is a frame that moves with the Sun in its orbit around the Milky Way galaxy but does not share its rotation. It is used in interplanetary tracking of planets and space crafts as they traverse the solar system. Further, using this interplanetary tracking technology with signals transmitted from Earth and reflected off a suitable planet, a new technique was developed that detects the orbital motion of the Earth through a solar-bound ether.

In this paper we review these modern tests for ether drift that confirm the existence of an Earth-bound ether first detected by Sagnac and later by Michelson and Gale. We also discuss a new interplanetary test that detects the existence of a solar-bound ether which Michelson and Morley tried unsuccessfully to detect.

\section{Detection of Ether Drift Arising from Rotation of the Earth}

We first review several tests using the GPS that detect ether drift resulting from the rotational motion of the Earth. This is exactly the motion that the Michelson-Gale experiment successfully detected in 1925 and which we argue revealed an Earth-bound ether that corresponds to the modern ECI frame.

\subsection{Detection Using GPS Clocks (Gift, 2010b; 2013)}

The availability of synchronized clocks in the GPS means that they can be used to determine the one-way speed of light by timing the transmission of a light signal travelling between two fixed points on the surface of the Earth. Consider therefore a clock A located a distance $l$ away from another clock B at the same latitude as shown in figure 1, clock B being East of clock A and both fixed on the surface of the Earth. (If necessary the distance between the clocks can be kept short in order to approximate an inertial frame and thereby negate any objections regarding the curvature of the Earth's surface and the associated non-inertial effects which are never raised in Michelson-Morley type experiments!) 


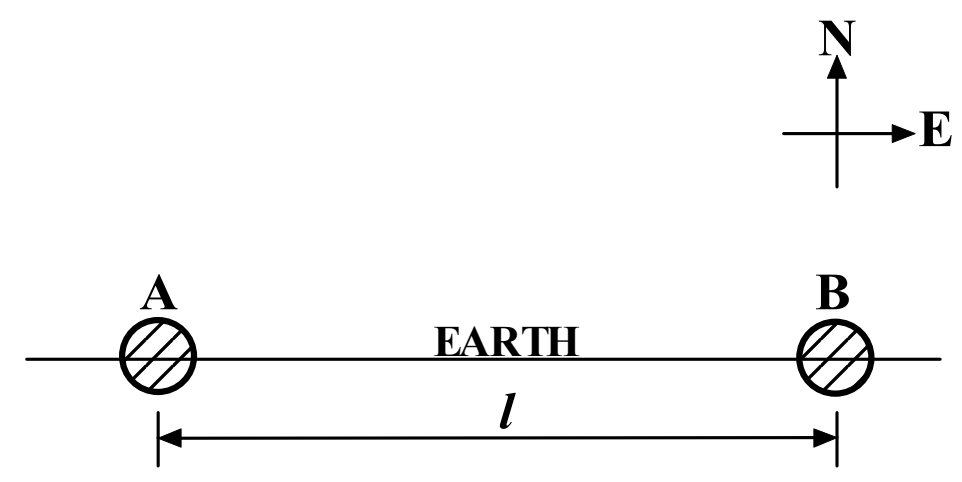

Figure 1. GPS Clocks A and B at fixed positions on Earth

\subsubsection{Eastward Transmission}

The CCIR clock synchronization rules give the total time $\Delta t$ for light to travel eastward along the path from clock A to clock B as (IS-GPS-200E, 2020; Ashby, 2003)

$$
\Delta t=\int_{\text {path }} \frac{d \sigma^{\prime}}{c}+\frac{2 \omega_{E}}{c^{2}} \int_{\text {path }} d A_{z}^{\prime}
$$

where $c$ is the speed of light, $d \sigma^{\prime}$ is infinitesimal distance in the moving frame, $\omega_{E}$ is the angular velocity of the rotating Earth and $d A_{z}^{\prime}$ is the infinitesimal area in the rotating coordinate system swept out by a vector from the rotation axis to the light pulse and projected onto a plane parallel to the equatorial plane. Performing the integration required in (1) yields (Gift, 2010; 2013)

$$
\Delta t=\frac{l}{c}+2 A_{z}^{\prime} \frac{\omega_{E}}{c^{2}}
$$

where $l$ is the distance between the two clocks both moving at speed $v$ the speed of the Earth's surface at that latitude. Let the circumference of the Earth at that latitude be $l_{C}$ and let the corresponding radius be $r$. Then the area $A_{z}^{\prime}$ is given by

$$
A_{z}^{\prime}=\frac{l}{l_{C}} \pi r^{2}
$$

Since $\omega_{E}=v / r$ and $l_{C}=2 \pi r$, substituting equation (3) in (2) results in

$$
\Delta t=\frac{l}{c}+\frac{l v}{c^{2}}
$$

The time interval $\Delta t$ given by (4) indicates the time for light to travel eastward between two points at the same latitude fixed on the surface of the Earth a distance $l$ apart and represents that determined using synchronized atomic clocks.

This elapsed time can be used to determine light speed between the two clocks. Since the distance between the two 
clocks is $l$, it follows that the one-way speed of light $c_{A B}$ traveling eastward between the two clocks is given by

$$
c_{A B}=\frac{l}{\Delta t}=\frac{l}{\frac{l}{c}+\frac{l v}{c^{2}}}=c\left(1+\frac{v}{c}\right)^{-1}=c\left(1-\frac{v}{c}+o\left(\frac{v}{c}\right)\right)=c-v, v<<c
$$

Thus, the synchronized clocks of the GPS give a one-way eastward light speed measurement of $c_{A B}=c-v$ relative to the surface of the Earth as deduced in the Michelson-Gale experiment of 1923.

\subsubsection{Westward Transmission}

For westward transmission again appropriately performing the integration in (1) yields the total time $\Delta t$ for light to travel the path westward from clock B to clock A given by (Gift, 2010; 2013)

$$
\Delta t=\frac{l}{c}-2 A_{z}^{\prime} \frac{\omega_{E}}{c^{2}}
$$

which reduces to

$$
\Delta t=\frac{l}{c}-\frac{l v}{c^{2}}
$$

The time interval $\Delta t$ given by (7) indicates the time for light to travel westward between two points at the same latitude fixed on the surface of the Earth a distance $l$ apart and represents that determined using synchronized atomic clocks.

Using the time found in (7) for one-way light travel in the westward direction, since the distance between the two clocks is $l$, it follows that the one-way speed of light $c_{B A}$ traveling westward between the two clocks is given by

$$
c_{B A}=\frac{l}{\Delta t}=\frac{l}{\frac{l}{c}-\frac{l v}{c^{2}}}=c\left(1-\frac{v}{c}\right)^{-1}=c\left(1+\frac{v}{c}+o\left(\frac{v}{c}\right)\right)=c+v, v<<c
$$

Thus, the synchronized clocks of the GPS give a one-way westward light speed measurement of $c_{B A}=c+v$ relative to the surface of the Earth again as indirectly confirmed in the Michelson-Gale experiment of 1923.

The results in (5) and (8) indicate that light speed is not the same in all directions as suggested by the null Michelson-Morey test. These results confirm the independent claims of Marmet and Kelley that light travels faster West than East relative to the surface of the rotating Earth. Specifically, the one-way measurement of light speed using GPS data in (5) directly indicates that a light signal sent eastward travels at speed $c$ minus the rotational speed of the Earth $v$ at that latitude $(c-v)$. The GPS data available in (8) also shows that a light signal sent westward travels at speed $c$ plus the rotational speed of the Earth $v$ at that latitude $(c+v)$. These generalized results were first published by Gift [34]. They directly confirm the indirect results of the Michelson-Gale experiments that revealed ether drift associated with the rotation of the Earth and thereby reveal the existence of an Earth-bound ether corresponding to the ECI frame.

\subsection{Michelson-Morley Experiment Using the GPS Clocks (Gift, 2012)}

With the availability of accurate synchronized clocks, a modified Michelson-Morley experiment can be conducted with direct timing of the signals traversing the orthogonal arms of the apparatus with no reliance on second-order interferometry. Such an approach was previously considered but never executed because of insufficient timing resolution (Sardin, 2004). The proposed approach does not encounter this problem since the light travel time is directly available from the GPS clock synchronization algorithm adopted by the CCIR (Kelly, 2005).

The basic configuration of the original Michelson-Morley experiment (Michelson \& Morley, 1887) is shown in 
figure 2 where the apparatus is moving with velocity $v$ through the hypothesized ether in direction PM1. Light from a source $\mathrm{S}$ splits into two beams at beam-splitter P. One beam travels from $\mathrm{P}$ to mirror M1 and back and is reflected at $\mathrm{P}$ into the interferometer $\mathrm{I}$. The second beam is reflected at $\mathrm{P}$ to mirror $\mathrm{M} 2$ and back and passes through $\mathrm{P}$ into the interferometer I where both beams form an interference pattern.

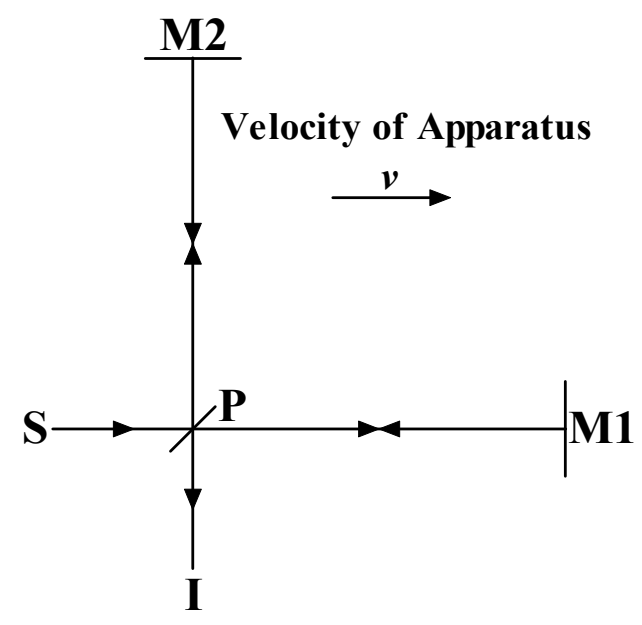

Figure 2. Michelson-Morley Experiment

In the frame of the moving apparatus as a result of ether drift, the resultant light speed between P and M1 would be $c-v$ toward M1 and $c+v$ toward $\mathrm{P}$ while the resultant light speed between $\mathrm{P}$ and M2 would be $\left(c^{2}-v^{2}\right)^{1 / 2}$ in both directions. For optical path lengths $P M 1=l_{1}$ and $P M 2=l_{2}$ the time $t_{1}(a)$ for the light to travel from P to M1 is given by

$$
t_{1}(a)=\frac{l_{1}}{c-v}
$$

and the time $t_{1}(b)$ for the light to travel from $\mathrm{M} 1$ to $\mathrm{P}$ is given by

$$
t_{1}(b)=\frac{l_{1}}{c+v}
$$

The time $t_{2}(a)$ for the light to travel from $\mathrm{P}$ to $\mathrm{M} 2$ is given by

$$
t_{2}(a)=\frac{l_{2}}{\sqrt{c^{2}-v^{2}}}
$$

and the time $t_{2}(b)$ for the light to travel from $\mathrm{M} 2$ to $\mathrm{P}$ is given by

$$
t_{2}(b)=\frac{l_{2}}{\sqrt{c^{2}-v^{2}}}
$$

The synchronized clocks in the GPS are now used to directly determine one-way light travel time. Thus in a modification of the original Michelson-Morley apparatus GPS clocks are placed at P, M1 and M2 in fig.2. 
Additionally, the arm PM1 is oriented along a line of latitude and the arm PM2 is positioned along a line of longitude. As a result of the rotation of the Earth there is movement of the apparatus at velocity $v=w$ in the direction PM1 towards the East where $w$ is the rotational speed of the surface of the Earth at the particular latitude.

2.2.1 Time Measurement along PM1

The time $t_{1}(a)_{G P S}$ measured by the GPS clocks at P and M1 for the light to travel from P to M1 is [34, 35]

$$
t_{1}(a)_{G P S}=\frac{l_{1}}{c}+\frac{l_{1} w}{c^{2}}
$$

while from equation (9) of ether theory

$$
t_{1}(a)=\frac{l_{1}}{c-w} \approx \frac{l_{1}}{c}+\frac{l_{1} w}{c^{2}}, w<<c
$$

Hence $t_{1}(a)_{G P S}=t_{1}(a)$ and ether drift arising from the rotation of the Earth is detected. The time $t_{1}(b)_{G P S}$ measured by the GPS clocks for the light to travel from M1 to P is (Gift, 2010; 2013)

$$
t_{1}(b)_{G P S}=\frac{l_{1}}{c}-\frac{l_{1} w}{c^{2}}
$$

while from equation (10) of ether theory

$$
t_{1}(b)=\frac{l_{1}}{c+w} \approx \frac{l_{1}}{c}-\frac{l_{1} w}{c^{2}}, w<<c
$$

Hence $t_{1}(b)_{G P S}=t_{1}(b)$ and ether drift arising from the rotation of the Earth is again detected. From ether theory as well as clock measurement, the difference in the out and back times along PM1 is given by

$$
\Delta t_{1}=t_{1}(a)-t_{1}(b)=\frac{2 l_{1}}{c} \frac{w}{c}
$$

Result (17) is first-order and therefore not affected by second-order effects such as length contraction as is the second-order result in the conventional Michelson-Morley type experiments. Equation (17) has been extensively verified in GPS operation.

2.2.2 Time Measurement along PM2

The time $t_{2}(a)_{G P S}$ for the light to travel from P to M2 measured by the GPS clocks at P and M2 is (Gift,

$$
t_{2}(a)_{G P S}=\frac{l_{2}}{c}
$$

while from equation (11) of ether theory

$$
t_{2}(a)=\frac{l_{2}}{\sqrt{c^{2}-w^{2}}} \approx \frac{l_{2}}{c}, w<<c
$$


Hence $t_{2}(a)_{G P S}=t_{2}(a)$ and ether theory is confirmed by GPS measurement. The time $t_{2}(b)_{G P S}$ for the light to travel from M2 to P measured by the GPS clocks is (Gift, 2013)

$$
t_{2}(b)_{G P S}=\frac{l_{2}}{c}
$$

while from equation (12) of ether theory

$$
t_{2}(b)=\frac{l_{2}}{\sqrt{c^{2}-w^{2}}} \approx \frac{l_{2}}{c}, w<<c
$$

Hence $t_{2}(b)_{G P S}=t_{2}(b)$ and ether theory is again confirmed by GPS measurement. From ether theory as well as GPS clock measurement, the difference in the out and back times along PM2 is given by

$$
\Delta t_{2}=t_{2}(a)-t_{2}(b)=0
$$

This has been confirmed by actual GPS measurements which have shown that unlike East-West travel, there is no time difference between light travelling North and light travelling South.

The modified Michelson-Morley experiment using synchronized GPS clocks to measure light travel times out and back along the arms of the apparatus has detected ether drift resulting from the rotation of the Earth. The clocks have directly confirmed the light travel times for changed light speeds $c \pm w$ in the East-West direction arising from the drift of the ether as the apparatus moves through the medium at speed $w$ corresponding to the speed of rotation of the Earth's surface at the particular latitude. They also directly confirm the indirect results of the Michelson-Gale experiments that revealed ether drift associated with the rotation of the Earth and once again reveal the existence of an Earth-bound ether corresponding to the ECI frame.

\subsection{Detection Using the GPS Range Equation (Gift, 2011)}

In section 2.1 the synchronized clocks of the GPS were used to determine travel time for light transmission between two fixed points on the same latitude of the Earth. Here the range equation used in the GPS to determine position on or close to the surface of the Earth is employed in the determination of travel time. Specifically, by substituting known spatial positions in the range equation, light travel times can be determined without the direct use of the synchronized clocks. These times can then be used to determine one-way light speed in the East-West direction.

The range equation is a central feature of the operation of the GPS. It represents light travel in the ECI frame which moves with the Earth as it revolves around the Sun but does not share the Earth's rotation. It is given by (IS-GPS-200E, 2010; Ashby, 2003)

$$
\left|\overline{r_{r}}\left(t_{r}\right)-\overline{r_{s}}\left(t_{s}\right)\right|=c\left(t_{r}-t_{s}\right)
$$

where $t_{s}$ is the time of transmission of an electromagnetic signal from a source, $t_{r}$ is the time of reception of the electromagnetic signal by a receiver, $\bar{r}_{s}\left(t_{s}\right)$ is the position of the source at the time of transmission of the signal and $\bar{r}_{r}\left(t_{r}\right)$ is the position of the receiver at the time of reception of the signal. Using GPS time measurements in equation (23) the position on the surface of the Earth can be accurately determined. This equation has been extensively tested and rigorously verified and has enabled the worldwide proliferation of the GPS. 
Wang (Wang, 2000) has used the range equation to show that the speed of light is dependent on the observer's uniform motion relative to the ECI frame. We use the range equation (23) of the GPS to determine elapsed time for light traveling between two points at the same latitude fixed at known positions on the surface of the rotating Earth. We then use this elapsed time and the known distance between the two fixed points to determine the one-way speed of light.

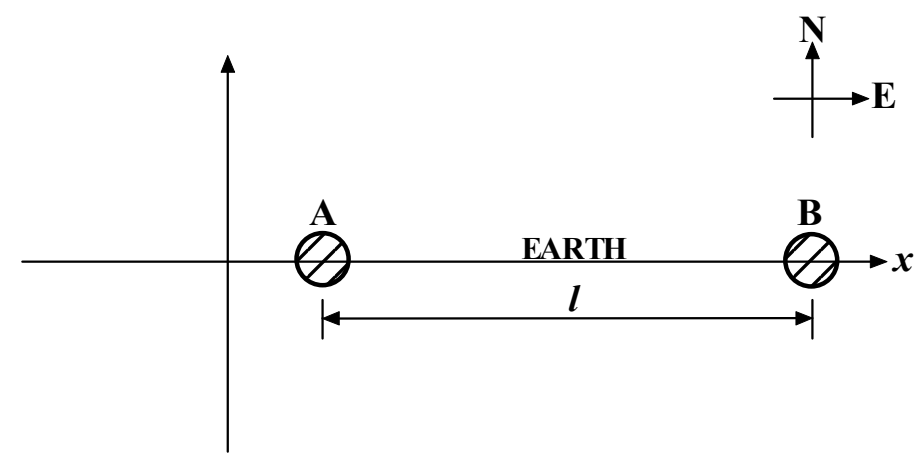

Figure 3. GPS Stations A and B at fixed positions on Earth

Consider figure 3 in which two GPS stations A and B are at the same latitude and fixed on the surface of the Earth a distance $l$ apart with B East of A. Since the Earth is rotating, the stations are moving eastward at speed $v$ the Earth's surface speed at the particular latitude. Let $l$ be sufficiently small such that the stations are moving uniformly in the same direction at speed $v$ relative to the ECI frame. In such circumstances stations A and B constitute an approximately inertial frame moving at speed $v$ relative to the ECI frame, a situation similar to the many light speed experiments conducted to test light speed constancy.

\subsubsection{Eastward Transmission}

Let station A transmit a signal eastward at time $t_{I}$ to station $\mathrm{B}$ which receives it at time $t_{F}$. On an axis fixed in the

ECI frame along the line joining the two stations with the origin west of station A, let $x_{A}(t)$ be the position of

station A at time $t$ and $x_{B}(t)$ be the position of station B at time $t$. Then from the range equation (23),

$$
x_{B}\left(t_{F}\right)-x_{A}\left(t_{I}\right)=c\left(t_{F}-t_{I}\right)
$$

where $x_{B}\left(t_{F}\right)$ is the position of station $\mathrm{B}$ at time $t_{F}$ and $x_{A}\left(t_{I}\right)$ is the position of station A at time $t_{I}$. Since the stations are moving uniformly in the same direction at speed $v$ relative to the ECI frame, it follows that the relation between the position $x_{B}\left(t_{F}\right)$ of station $\mathrm{B}$ at the time of reception of the signal and its position $x_{B}\left(t_{I}\right)$ at the time of emission of the signal is given by

$$
x_{B}\left(t_{F}\right)=x_{B}\left(t_{I}\right)+v\left(t_{F}-t_{I}\right)
$$

Substituting for $x_{B}\left(t_{F}\right)$ from (25) in (24) yields

$$
x_{B}\left(t_{I}\right)-x_{A}\left(t_{I}\right)+v\left(t_{F}-t_{I}\right)=c\left(t_{F}-t_{I}\right)
$$

This gives the elapsed time as 


$$
\left(t_{F}-t_{I}\right)=\frac{l}{c-v}
$$

Therefore, the speed $c_{A B}$ of the light traveling from station A to station $\mathrm{B}$ is given by separation $l$ divided by elapsed time $\left(t_{F}-t_{I}\right)$ which using $(27)$ is

$$
c_{A B}=\frac{l}{\left(t_{F}-t_{I}\right)}=\frac{l}{l /(c-v)}=c-v
$$

\subsubsection{Westward Transmission}

Let station B transmit a signal westward at time $t_{I}$ to station A which receives it at time $t_{F}$. Then using the range equation (23) and noting that $x_{B}\left(t_{I}\right)>x_{A}\left(t_{F}\right)$,

$$
x_{B}\left(t_{I}\right)-x_{A}\left(t_{F}\right)=c\left(t_{F}-t_{I}\right)
$$

where $x_{B}\left(t_{I}\right)$ is the position of station B at time $t_{I}$ and $x_{A}\left(t_{F}\right)$ is the position of station A at time $t_{F}$. Since the stations are moving uniformly in the same direction at speed $v$ relative to the ECI frame, the relation between the position $x_{A}\left(t_{F}\right)$ of station $\mathrm{A}$ at the time of reception of the signal and its position $x_{A}\left(t_{I}\right)$ at the time of emission of the signal is given by

$$
x_{A}\left(t_{F}\right)=x_{A}\left(t_{I}\right)+v\left(t_{F}-t_{I}\right)
$$

Substituting for $x_{A}\left(t_{F}\right)$ from (30) in (29) yields

$$
x_{B}\left(t_{I}\right)-x_{A}\left(t_{I}\right)-v\left(t_{F}-t_{I}\right)=c\left(t_{F}-t_{I}\right)
$$

This yields the elapsed time as

$$
\left(t_{F}-t_{I}\right)=\frac{l}{c+v}
$$

Therefore, the speed $c_{B A}$ of the light traveling from station $\mathrm{B}$ to station $\mathrm{A}$ is given by separation $l$ divided by elapsed time $\left(t_{F}-t_{I}\right)$ which using (32) is

$$
c_{B A}=\frac{l}{\left(t_{F}-t_{I}\right)}=\frac{l}{l /(c+v)}=c+v
$$

The results in equations (28) and (33) first reported in (Gift, 2011) indicate that light travels faster West than East relative to the surface of the Earth. To be specific, the one-way determination of light speed using the range equation of the GPS establishes in (28) that a signal sent eastward travels at speed $c$ minus the rotational speed of the Earth $v$ at that latitude giving $c-v$. The range equation also shows in (33) that a signal sent westward travels at speed $c$ plus the rotational speed of the Earth $v$ at that latitude giving $c+v$. This is true for short-distance travel involving an approximately inertial frame and also long-distance circumnavigation of the Earth. This again completely corroborates the light speed determined using synchronized GPS clocks. It is also consistent with the Michelson-Gale experiment and confirms the detection of ether drift arising from the Earth's rotation thereby confirming the existence of an Earth-bound ether which is the ECI frame. 


\subsection{Detection Using GPS Time Transfer (Gift, 2014a)}

Time Transfer is the process of communicating time information using electromagnetic signal transmission through space. It is used for example to maintain coordination of time and frequency in systems operating at or close to the Earth and beyond. The method is based on an algorithm published by the International Telecommunications Union (ITU) that has been rigorously tested and completely verified (ITU, 2012). It involves the transmission of a signal from one GPS station to another such that system synchronization can be effected. Today it is part of the standard procedure employed in time comparisons between separated laboratories on the rotating Earth and is employed in a range of applications (Nelson, 2011; Ashby, 2010; Petit \& Wolf, 2005; Blanchet et al., 2001).

The approach described in the ITU recommendation (ITU, 2012) is to use electromagnetic signal transmission in the Earth-Centred Inertial (ECI) frame where it travels at speed $c$ to determine the travel time from a satellite to a ground receiver which is moving at speed $v$, the rotational speed of the Earth at the particular latitude. Because light speed in the ECI frame is known, the determination of the transfer time $\Delta t$ is essentially straightforward. Consider an electromagnetic pulse transmitted from a geo-stationary GPS satellite at position $\bar{r}_{T}$ and GPS time $t_{T}$ travelling at speed $c$ relative to the ECI frame to a ground receiver whose position at GPS time $t_{T}$ is $\bar{r}_{R}$ and whose velocity because of the rotation of the Earth is $v$ relative to the ECI frame. By utilizing a geo-stationary satellite, the test is fully within the frame of the rotating Earth as are the previous tests done with synchronized clocks and the range equation. Let $\theta$ be the angle between the direction of propagation of the signal and $v$ which, because $v<<c$ can be represented as shown in fig.4. Here $R$ is the initial distance between the geo-stationary satellite and the receiver. If the signal arrives at the receiver at time $t_{R}$ then for the signal transmission interval $\Delta t=t_{R}-t_{T}$ the receiver experiences a displacement $\bar{v} \Delta t$.

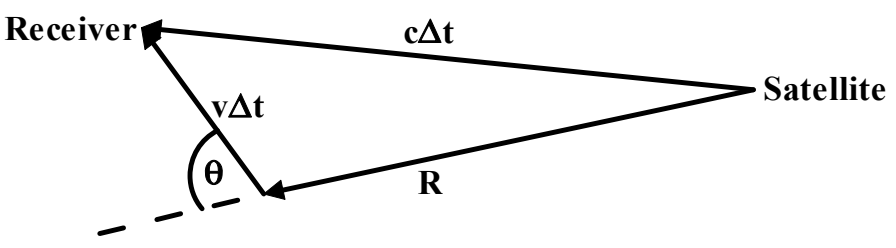

Figure 4. Light Transmission from Geo-stationary Satellite to Earth-based Receiver

For signal travel within the ECI frame from satellite to receiver at speed $c$ in time $\Delta t$, the signal displacement $c \Delta t$ is given by

$$
c \Delta t=\bar{R}+\bar{v} \Delta t
$$

where $\bar{R}=\bar{r}_{R}-\bar{r}_{T}$. Solving equation (34) for $\Delta t$ yields the transfer time (Ashby, 2010)

$$
\Delta t=\frac{R}{c}+\frac{\bar{v} \cdot \bar{R}}{c^{2}}
$$

The transfer time $\Delta t$ in (35) for light travelling from the orbiting satellite to a ground-based receiver has been fully tested and experimentally verified and is routinely used in time comparisons. It is therefore accurate and can be used to evaluate the light speed $c_{R}$ relative to the ground receiver. Using the distance $R$ between the satellite and the receiver at the time of transmission of the signal, light speed $c_{R}$ is given by

$$
c_{R}=\frac{R}{\Delta t}=\frac{R}{\frac{R}{c}+\frac{\bar{v} \cdot \bar{R}}{c^{2}}}
$$

But from Figure 4, $\bar{v} \cdot \bar{R}=v R \cos \theta$ and hence (36) becomes 


$$
c_{R}=\frac{R}{\frac{R}{c}+\frac{v R \cos \theta}{c^{2}}}=\frac{c^{2}}{c+v \cos \theta}=\frac{c^{2}(c-v \cos \theta)}{c^{2}-v^{2} \cos ^{2} \theta} \approx c-v \cos \theta, v<<c
$$

Therefore using the experimentally confirmed transfer time $\Delta t$ and the initial separation $R$ give a light speed $c_{R}=c-v \cos \theta$ relative to the receiving station and not $c_{R}=c$ as presented in the ITU recommendation and required by special relativity. For movement of the receiving station away from the satellite, $\theta=0^{\circ}$ and therefore (37) gives $c_{R}=c-v$. For movement of the receiving station toward the satellite, $\theta=180^{\circ}$ and hence (37) yields $c_{R}=c+v$. These light speed values using signal transmission from a GPS satellite to a ground-based receiver have also been observed by Sato (Sato, 2010) and are exactly those found using synchronous clocks and the range equation. To further demonstrate the correctness of the light speed $c_{R}=c-v \cos \theta$, the transfer time $\Delta t$ is calculated using this speed. Thus

$$
\Delta t=\frac{R}{c_{R}}=\frac{R}{c-v \cos \theta}=R \frac{c+v \cos \theta}{c^{2}-v^{2} \cos ^{2} \theta}=\frac{R}{c^{2}}(c+v \cos \theta), v<<c
$$

Equation (38) gives

$$
\Delta t=\frac{R}{c}+\frac{R v \cos \theta}{c^{2}}=\frac{R}{c}+\frac{\bar{v} \cdot \bar{R}}{c^{2}}
$$

which is exactly the transfer time (35) given in the ITU recommendations that has been rigorously tested and confirmed. Thus, the direct detection of the rotational motion of the Earth has again been achieved and an Earth-bound ether or the ECI frame has again been detected within the frame of the rotating Earth.

\section{Detection of the Orbital Motion of the Earth Using Modern Technology (Gift, 2014b)}

Having successfully confirmed the existence of an Earth-bound ether in the form of the ECI frame through detection of the rotational motion of the Earth, we turn now to detection of orbital motion of the Earth within the frame of the orbiting Earth. While positions on the surface of the Earth can be accurately determined using the range equation of the GPS referred to the ECI frame, the orbital ephemerides of the planets and other bodies in the solar system are determined using a different set of equations operating within the sun-centered inertial or solar-system barycentric frame (Aleksandrov et al., 1972; Ostro, 1993; Myles Standish \& Williams, accessed November 11, 2020). The solar-system barycentric or Sun-Centered Inertial (SCI) frame is a frame that moves with the Sun but does not rotate with it and provides a convenient reference frame for a range of astronomical events. Two equations which have been empirically determined are used to determine round-trip time of an electromagnetic signal that emanates from a transmitting antenna on Earth and is reflected by a planetary body, spacecraft transponder or satellite back to the same antenna on Earth. Time measurement is accomplished using accurate atomic clocks based on Coordinated Universal Time (UTC) and the spatial coordinates are taken relative to the solar-system barycenter.

The specific equations are given by (Aleksandrov et al., 1972; Ostro, 1993; Myles Standish \& Williams, accessed November 11, 2020)

$$
\begin{gathered}
c \tau_{u}=\left|r_{B}\left(t_{R}-\tau_{d}\right)-r_{A}\left(t_{R}-\tau_{d}-\tau_{u}\right)\right| \\
c \tau_{d}=\left|r_{A}\left(t_{R}\right)-r_{B}\left(t_{R}-\tau_{d}\right)\right|
\end{gathered}
$$

where $t_{R}$ is the time of reception of the signal, $\tau_{u}$ and $\tau_{d}$ are the up-leg and down-leg times respectively, $r_{A}$ is the solar-system barycentric position of the receiving antenna on the Earth's surface, $r_{B}$ is the solar-system barycentric position of the reflector which is either a responding spacecraft or the reflection point on the planet's surface and $c$ is 
the speed of light in the solar-system barycentric frame. These equations are based on the observation that light travels in the SCI frame at a constant speed $c$ and have been exhaustively tested and rigorously verified. They are derived using data sets collected over a period of more than half a century of measurement. The two equations are solved iteratively in order to obtain values for $\tau_{u}$ and $\tau_{d}$. In practice time corrections must be made to $\tau_{u}$ and $\tau_{d}$ because of relativistic effects, the electron content of the solar corona and the Earth's troposphere (Ostro, 1993; Myles Standish \& Williams, accessed November 11, 2020).

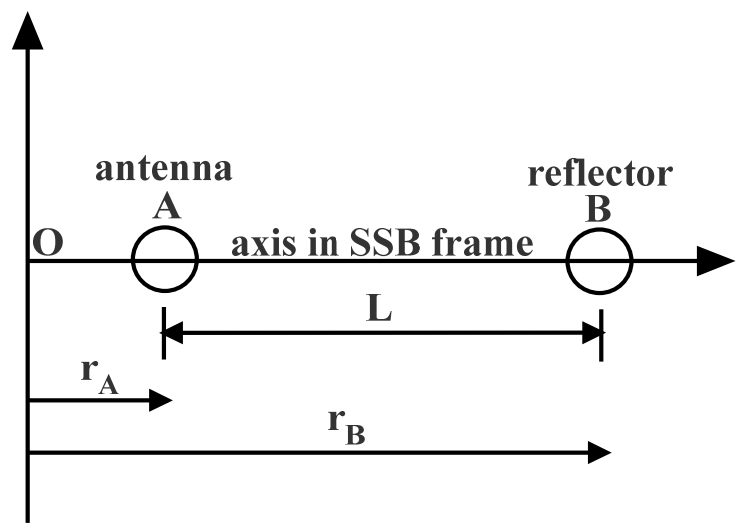

Figure 6. Antenna A and Reflector B at instant of Light Reflection

In a test to detect ether drift resulting from the orbital motion of the Earth, this system is used to determine one-way light speed for light from elsewhere in the solar system that is detected on the orbiting Earth. We consider a transmitting-receiving antenna A fixed on the surface of the Earth which is moving in the SCI frame at orbital speed $v$ relative to this frame and a reflecting satellite B stationary or moving relative to the SCI frame beyond the boundaries of the Earth-bound ether. On an axis fixed in the SCI frame along the line joining antenna A and satellite B at the instant of signal reflection with antenna $\mathrm{A}$ closer to the origin $\mathrm{O}$ than satellite $\mathrm{B}$ and taking positive values, let $r_{A}$ be the coordinate along the axis of the position in the SSB frame of antenna A and $r_{B}$ be the coordinate along the axis of the position of satellite $\mathrm{B}$. At the time of reflection of the signal from reflector $\mathrm{B}$, let the distance between $\mathrm{A}$ and $\mathrm{B}$ be $L$ given by

$$
r_{B}\left(t_{R}-\tau_{d}\right)-r_{A}\left(t_{R}-\tau_{d}\right)=L
$$

\subsection{Antenna Moving toward Reflector}

At the instant of reflection of the electromagnetic signal at reflector B let antenna A move directly toward the reflector $\mathrm{B}$ at orbital speed $v$ relative to the SCI frame. Then using equation (42),

$$
c \tau_{d}=r_{B}\left(t_{R}-\tau_{d}\right)-r_{A}\left(t_{R}\right)
$$

Since (for sufficiently small $L$ ) antenna A is moving uniformly toward reflector B at speed $v$ relative to the SCI frame, it follows that the relation between the position $r_{A}\left(t_{R}\right)$ of antenna $\mathrm{A}$ at the time of reception of the signal and its position $r_{A}\left(t_{R}-\tau_{d}\right)$ at the time of reflection of the signal at reflector $\mathrm{B}$ is given by

$$
r_{A}\left(t_{R}\right)=r_{A}\left(t_{R}-\tau_{d}\right)+\tau_{d} v
$$

Substituting for $r_{A}\left(t_{R}\right)$ from (45) in (44) yields 


$$
\left.c \tau_{d}=r_{B}\left(t_{R}-\tau_{d}\right)-r_{A}\left(t_{R}-\tau_{d}\right)\right)-\tau_{d} v
$$

Using (43) this becomes

$$
r_{B}\left(t_{R}-\tau_{d}\right)-r_{A}\left(t_{R}-\tau_{d}\right)=L=(c+v) \tau_{d}
$$

Hence for an observer on Earth equation (42) yields the down-leg time $\tau_{d}$ as

$$
\tau_{d}=\frac{L}{c+v}
$$

Therefore the speed $c_{B A}$ of the electromagnetic signal relative to antenna A traveling from the reflector $\mathrm{B}$ to the moving antenna $\mathrm{A}$ is given by the separation $L$ at the time of reflection divided by the down-leg time $\tau_{d}$ which using $(48)$ is

$$
c_{B A}=\frac{L}{\tau_{d}}=\frac{L}{L /(c+v)}=c+v
$$

\subsection{Antenna Moving away from Reflector}

At the instant of reflection of the electromagnetic signal at reflector B let antenna A move directly away from the reflector $\mathrm{B}$ at orbital speed $v$ relative to the SCI frame. Then using equation (42),

$$
c \tau_{d}=r_{B}\left(t_{R}-\tau_{d}\right)-r_{A}\left(t_{R}\right)
$$

Since antenna A is moving uniformly away from reflector B at speed $v$ relative to the SSB frame, it follows that the relation between the position $r_{A}\left(t_{R}\right)$ of antenna $\mathrm{A}$ at the time of reception of the signal and its position $r_{A}\left(t_{R}-\tau_{d}\right)$ at the time of reflection of the signal at reflector $\mathrm{B}$ is given by

$$
r_{A}\left(t_{R}\right)=r_{A}\left(t_{R}-\tau_{d}\right)-\tau_{d} v
$$

Substituting for $r_{A}\left(t_{R}\right)$ from (51) in (50) yields

$$
\left.c \tau_{d}=r_{B}\left(t_{R}-\tau_{d}\right)-r_{A}\left(t_{R}-\tau_{d}\right)\right)+\tau_{d} v
$$

Using (43) this becomes

$$
r_{B}\left(t_{R}-\tau_{d}\right)-r_{A}\left(t_{R}-\tau_{d}\right)=L=(c-v) \tau_{d}
$$

Hence for an observer on Earth the range equation (42) yields the down-leg time $\tau_{d}$ as

$$
\tau_{d}=\frac{L}{c-v}
$$

Therefore, the speed $c_{B A}$ of the electromagnetic signal relative to antenna A traveling from the reflector B to the moving antenna $\mathrm{A}$ is given by the separation $L$ at the time of reflection divided by the down-leg time $\tau_{d}$ which using (54) is

$$
c_{B A}=\frac{L}{\tau_{d}}=\frac{L}{L /(c-v)}=c-v
$$


It follows from (49) and (55) that light travels from the reflector to the antenna on Earth at a speed $c+v$ relative to the antenna in the case of the Earth moving toward the reflector at orbital speed $v$ and light travels at speed $c-v$ relative to the antenna in the case of the Earth moving away from the reflector at orbital speed $v$. Thus, light speed variation for light travelling in the SCI frame has been detected and agrees with findings of Wallace (Wallace, 1969) for light travel through space. This represents detection of a Sun-bound ether as a medium of light propagation through which the Earth is moving as it orbits the Sun and in which light travels at constant speed $c$. This Sun-bound ether drift detection of the orbital motion of the Earth was first reported by Gift using the Doppler and Roemer effects (Gift, 2006) involving light sources external to the Earth and is exactly the SCI frame in which light travels at speed $c$.

If the velocity of the reflecting satellite B is made such that it is geostationary, then the receiving antenna and reflector are fixed relative to each other and to the surface of the moving Earth. In such a case the complete light speed determination yielding $c \pm v$ is in the frame of the moving Earth. These results in the SCI frame indicate that the orbital speed $v$ of the uniformly moving Earth is readily detectable with apparatus in which the signal source and receiver are fixed relative to each other and to the moving Earth.

These results represent detection of a solar-bound ether that corresponds to the SCI frame which supports light travel at speed $c$. This is similar to the Earth-bound ether detected earlier that corresponds to the ECI frame which also supports light travel at speed c. Thus, while Michelson and Morley failed to detect ether drift arising from the Earth's orbital motion in their celebrated experiment of 1887 , modern technology in the form of an interplanetary tracking technique has enabled detection of this ether-drift.

\section{Discussion}

The four techniques utilizing the GPS discussed in this paper all detect ether drift associated with an Earth-bound ether or ECI frame (where light speed is $c$ ) arising from the Earth's rotation as evidenced by light speed changes $c \pm v$ for light travelling in the east-west direction. These techniques include the synchronized clocks of the GPS, a GPS-based Michelson-Morley experiment, the range equation of the GPS and Time Transfer technology, all within the frame of the rotating Earth. The detection of ether drift associated with a solar-bound ether or SCI frame (where light speed is also $c$ ) arising from the orbital motion of the Earth was also accomplished, in this case using interplanetary tracking technology within the frame of the orbiting Earth.

The observed light speed variation arising from rotational motion within the ECI frame and orbital motion within the SCI frame where light travels at a speed $c$ in either frame suggests the existence of an Earth-bound ether inside a larger solar-bound ether, which are carried along by the Earth and the Sun respectively (Sato, 2010; Su, 2001). Su (Su, 2001) has extensively developed this thesis within the framework of electromagnetic theory and has suggested the possible existence of a hierarchy of ethers where the Earth-bound ether is located within the solar-bound ether, which itself may be immersed in a galaxy-bound ether and so on. He stated, "It is supposed that an electromagnetic wave propagates via a medium like the ether. However, the ether is not universal. It is proposed that in the region under sufficient influence of the gravity due to the earth, the sun, or another celestial body, there forms a local ether which in turn is stationary with respect to the gravitational potential of the respective body. Thereupon, each local ether together with the gravitational potential moves with the associated celestial body. Thus, the earth and the sun local ethers are stationary in a geocentric and a heliocentric inertial frame, respectively. Consequently, the propagation of an earthbound electromagnetic wave is referred to this geocentric frame and is entirely independent of earth's orbital motion around the sun or whatever, while for interplanetary propagation, the sun local ether is the main propagation medium and hence the majority of propagations are referred to this heliocentric frame and independent of the orbital motion of the sun." In view of the evidence discussed in this paper, this proposal is a reasonable working hypothesis regarding the nature of the ether. Hatch (Hatch, 2004) however offered an interpretation in which the basic phenomenon is attributed to clock bias since the clocks are synchronized within the specific inertial frame in which they are operating. This phenomenon of multiple ethers with constant light speed within each local ether calls for further investigation in order to fully understand the nature of the medium and its underlying structure.

Notwithstanding this uncertainty, the measurement of light speed variation because of movement in the ECI frame and the SCI frame is irrefutable and is therefore experimental confirmation of the existence of an ether in the form of an Earth-bound ether or ECI frame located within a solar-bound ether or SCI frame where in both cases light speed is $c$. This existence of an ether as the medium of electromagnetic wave propagation with measurable the characteristics permittivity and permeability means that energy will be lost in the propagation process, lest there be perpetual 
motion. This corresponds to a small but non-zero conductivity $\sigma_{o}$ of space which Monti indicates has a value $\sigma_{o}=2.85 \times 10^{-29}(\Omega . m)^{-1}$ for a vacuum (Monti, 1988). Under such a condition, the solution to Maxwell's equations is a damped electromagnetic wave that loses energy as it propagates. From the law of the photoelectric effect according to which the energy of an electromagnetic wave is directly proportional to its frequency, as the travelling wave loses energy the frequency of the wave falls. This corresponds to an increase of the wave length from $\lambda_{o}$ at the time of emission to $\lambda_{1}$ after travelling a distance $r$ through space. The solution of Maxwell's equations leads to the relation (Monti, 1996)

$$
r=\frac{1}{R_{o} \sigma_{o}} \ln (z+1)
$$

where $r$ is the path length travelled by the damped electromagnetic wave, $R_{o}=\sqrt{\mu_{o} / \varepsilon_{o}}=377 \Omega$ is the wave resistance of the ether and $z=\left(\lambda_{1}-\lambda_{o}\right) / \lambda_{o}$ is photon redshift. Equation (56) can be rearranged to yield

$$
z(r)=e^{\beta r}-1
$$

where $\beta=R_{o} \sigma_{o}$. Equation (57) shows that the energy loss of the photon results in an exponential increase of the redshift with the distance travelled by the light. This is the tired-light model of photon redshift for light from distant galaxies. For astronomically short distances where $\beta r<<1$, equation (57) reduces to the linear relation

$$
z=\beta r
$$

Equation (58) is the well-known redshift-distance relation for galaxies known as Hubble's Law.

La Violette (La Violette, 1986) has shown that the full equation (57) representing tired light cosmology better fits the redshift-distance data for galaxies than big bang cosmology. He has shown that this is true for several other cosmological tests. With the confirmed existence of the ether, Hubble's law is therefore not an indication that the universe is expanding as is the accepted interpretation, but instead is a manifestation of energy loss as light travels long distances through the ether.

The existence of the ether has another major consequence. In the fifth edition of his book "Relativity: The Special and the General Theory" first published in 1916, Albert Einstein declared "But on the basis of the theory of relativity...there can be no aether-drift, nor any experiment with which to demonstrate it." Therefore, with the detection of the ether, the immediate collapse of special relativity and the associated Lorentz transformations follows. In search of correct space-time transformations, we refer to the important work of Franco Selleri. He developed a universal set of all possible space-time transformations connecting two inertial frames under a set of reasonable assumptions (Selleri, 1997; 2010). The unique value of this set is that it allows the determination of that set of linear space-time transformations which best accord with the physical world from among all the possible transformations. These members of the set have the same space transformation but have time transformations that differ by a clock synchronization parameter $e_{1}$ given by

$$
t^{\prime}=t / \gamma+e_{1}(x-v t)
$$


The Lorentz transformations of special relativity correspond to $e_{1}=-v \gamma / c^{2}$ while the Selleri transformations constituting an absolute space theory correspond to $e_{1}=0$. It is surprising that all these transformations make the same predictions for a broad range of phenomena including Michelson-Morley, Roemer and Doppler despite the synchronization difference (Selleri, 2004). They however predict different light speeds given by

$$
c\left(S^{\prime}\right)=\frac{c}{1+\beta+c e_{1} \sqrt{1-\beta^{2}}}, \beta=v / c
$$

where the clock synchronization parameter $e_{1}$ is evident. Selleri (Selleri, 2010; 2004; 2011) showed in several different ways that $e_{1}=0$ and this value of the parameter has been independently confirmed by Gift (Gift, 2015) using light speed analysis in an inertial frame. The transformations resulting from $e_{1}=0$ are referred to as the Selleri transformations. With $e_{1}=0$ in (60), these transformations predict light speed $c\left(S^{\prime}\right)=\frac{c}{1+\beta} \approx c-v, v<<c$, the light speed detected in the various tests discussed in this paper and not constant light speed $c$ predicted by the Lorentz transformations corresponding to $e_{1}=-v \gamma / c^{2}$ in (60).

\section{Conclusion}

Ether drift arising from the rotational and orbital motions of the Earth has been detected using a range of modern tests involving the GPS which were discussed in this paper. As in an earlier report (Gift, 2006), this detection was achieved through the agency of light speed variation resulting from movement through a local ether. One set of tests detect ether drift associated with the rotational motion of the Earth and confirm the existence of an Earth-bound ether which we identify as the ECI frame. These are first-order tests that give results that are consistent with the Michelson-Gale experiment, the first to detect ether drift resulting from the Earth's rotational motion. However, the Sagnac experiment was the first to detect ether drift associated with movement in the Earth-bound ether or ECI frame, and this was accomplished using rotating apparatus within a laboratory on the surface of the Earth.

A new test involving interplanetary tracking technology detected ether drift arising from the orbital motion of the Earth, a motion that was also detected using the Roemer and Doppler effects (Gift, 2006). This is the motion that Michelson and Morley unsuccessfully attempted to detect in their failed 1887 experiment. The new test, as well as the earlier Roemer and Doppler tests, succeeded in detecting a solar-bound ether now recognized as the SCI frame.

Thus, in view of the vast body of theoretical, experimental and observational evidence supporting light wave transmission through an all-pervasive medium (Whittaker, 1973; Schaffner, 1972), particularly the amazing discovery of electromagnetic waves with speed set by measurable characteristics of the ether medium, the positive detection of ether drift for rotational and orbital motion using GPS and other modern technology described in this paper represents a turning point in scientific development. It should hasten the rejection of the relativistic worldview and hopefully usher in a new and exciting period of research and discovery about the nature and properties of the ether.

\section{Conflict of interests}

The authors declare that there is no conflict of interests regarding the publication of this paper.

\section{References}

Aleksandrov, Y. N., Kuznetsov, B. I., Petrov, G. M., \& Rzhiga, O. N. (1972). Techniques of Radar Astrometry. Soviet Astronomy, 16(137), 1972. 
Allan, D. W., Weiss, M. A., \& Ashby, N. (1985). Around-the-World Relativistic Sagnac Experiment. Science, $228,69$. https://doi.org/10.1126/science.228.4695.69

Allen, C. O., Kiess, T. E., Nelson, R. A., Sergienko, A. V., Shih, Y. H., Wang, B. C., \& Yang, F. M. (1992). Plans to Improve the Experimental Limit in the Comparison of the East-West and West-East One-way Light Propagation Times on the Rotating Earth. Proceedings of the 24th PTTI Meeting, 105-109.

Ashby, N. (2003). Relativity in the Global Positioning System. Living Reviews in Relativity, 6, 1. https://doi.org/10.12942/lrr-2003-1

Ashby, N. (2010). The Sagnac Effect in the Global Positioning System. In G. Rizzi \& M. L. Ruggiero (Eds.), Relativity in Rotating Frames. Kluwer Academic Publishers, London.

Bethell, T. (2009). Questioning Einstein: Is Relativity Necessary? Vales Lake Publishing, Colorado.

Bilger, H. R., Stedman, G. E., Li, G., Schreiber, U., \& Schneider, M. (1995). Ring Lasers for Geodesy. IEEE Transactions on Instrumentation \& Measurement, 44, 468. https://doi.org/10.1109/19.377882

Blanchet, L., Salomon, C., Teyssandier, P., \& Wolf, P. (2001). Relativistic theory for time and frequency transfer to order c-3. Astronomy and Astrophysics, 370, 320. https://doi.org/10.1051/0004-6361:20010233

Brillet, A., \& Hall, J. L. (1979). Improved Laser Test of the Isotropy of Space. Physical Review Letters, $42,549$. https://doi.org/10.1103/PhysRevLett.42.549

Buchwald, D., \& Thorne, K. S. (2005). Preface to the New Edition. The Born-Einstein Letters 1916-1965 by M. Born, Macmillan, New York.

DeMeo, J. (2014). Does a Cosmic Ether Exist? Evidence from Dayton Miller and Others. Journal of Scientific Exploration, 28, 647.

Eisele, C., Nevsky, A., \& Schiller, S. (2009). Laboratory Test of the Isotropy of Light Propagation at the 10-17 Level. Physical Review Letters, 103, 090401. https://doi.org/10.1103/PhysRevLett.103.090401

Gift, S. J. G. (2006). The Relative Motion of the Earth and the Ether Detected. Journal of Scientific Exploration, 20, 201.

Gift, S. J. G. (2010a). Light Speed Invariance is a Remarkable Illusion. Physics Essays, $23,1$. https://doi.org/10.4006/1.3280803

Gift, S. J. G. (2010b). One-Way Light Speed Measurement Using the Synchronized Clocks of the Global Positioning System (GPS). Physics Essays, 23, 271. https://doi.org/10.4006/1.3361840

Gift, S. J. G. (2011). One-Way Light Speed Determination Using the Range Measurement Equation of the GPS. Applied Physics Research, 3, 110. https://doi.org/10.5539/apr.v3n1p110

Gift, S. J. G. (2012). Successful Search for Ether Drift in a Modified Michelson-Morley Experiment Using the GPS. Applied Physics Research, 4, 185. https://doi.org/10.5539/apr.v4n1p185

Gift, S. J. G. (2013). Light Transmission and the Sagnac Effect on the Rotating Earth. Applied Physics Research, 5 , 93. https://doi.org/10.5539/apr.v5n5p93

Gift, S. J. G. (2014a). One-Way Speed of Light Using Interplanetary Tracking Technology. Physical Science International Journal, 4, 780. https://doi.org/10.9734/PSIJ/2014/7783

Gift, S. J. G. (2014b). Time Transfer and the Sagnac Correction in the GPS. Applied Physics Research, 6, 1. https://doi.org/10.5539/apr.v6n6p1

Gift, S. J. G. (2015). The Selleri Transformations and the One-way Speed of Light. Physics Essays, $28,474$. https://doi.org/10.4006/0836-1398-28.4.474

Hatch, R. R. (2004). Those Scandalous Clocks. GPS Solutions, 8, 67. https://doi.org/10.1007/s10291-004-0092-8

Hermann, S., Senger, A., Kovalchuk, E., Müller, H., \& Peters, A. (2005). Test of the Isotropy of the Speed of Light Using a Continuously Rotating Optical Resonator. Physical Review Letters, 95, 150401. https://doi.org/10.1103/PhysRevLett.95.150401

International Telecommunication Union. (2012). Relativistic Time Transfer in the Vicinity of the Earth and in the Solar System, Recommendation ITU-R TF.2018, August.

IS-GPS-200E 8 June 2010. Retrieved from http://www.gps.gov/technical/icwg/IS-GPS-200E.pdf 
Kelly, A. (2005). Challenging Modern Physics. BrownWalker Press, Florida.

La Violette, P. A. (1986). Is the Universe Really Expanding? Astrophysical Journal, $301,544$. https://doi.org/10.1086/163922

Levy, J. (2003). From Galileo to Lorentz... and Beyond. Apeiron, Montreal.

Lorentz, H. A. (1953). Electromagnetic Phenomena in a System Moving with any Velocity less than that of Light. In H. A. Lorentz, A. Einstein, H. Minkowski, \& H. Weyl, (Eds.), The Principle of Relativity. Dover Publications, New York.

Mansouri, R, \& Sexl, R. U. (1977). A Test Theory of Special Relativity: II. First-Order Tests. General Relativity and Gravitation, 8, 515-524. https://doi.org/10.1007/BF00762635

Marmet, P. (2000). The GPS and the Constant Velocity of Light. Acta Scientiarum, 22, 1269.

Michelson, A. A. (1904). Relative Motion of Earth and Ether. Philosophical Magazine, $8,716$. https://doi.org/10.1080/14786440409463244

Michelson, A. A., \& Gale, H. E. (1925). The Effect of the Earth's Rotation on the Velocity of Light. Astrophysical Journal, 61, 140. https://doi.org/10.1086/142879

Michelson, A. A., \& Morley, E. W. (1887). The relative motion of the Earth and the luminiferous aether. American Journal of Science, ser.3(34), 333. https://doi.org/10.2475/ajs.s3-34.203.333

Miller, D. C. (1933). The ether-drift experiment and the determination of the absolute motion of the earth. Review of Modern Physics, 5, 203. https://doi.org/10.1103/RevModPhys.5.203

Monti, R. A. (1988). The Electric Conductivity of Background Space. In Problems in Quantum Physics (p. 640). World Scientific, Singapore.

Monti, R. A. (1996). Theory of Relativity: A Critical Analysis. Physics Essays, 9, 238. https://doi.org/10.4006/1.3029231

Müller, H., Stanwix, P. L., Tobar, M. E., Ivanov, E., Wolf, P., Herrmann, S., Senger, A., Kovalchuk, E., \& Peters, A. (2007). Relativity tests by complementary rotating Michelson-Morley experiments. Physical Review Letters, 99, 050401. https://doi.org/10.1103/PhysRevLett.99.050401

Myles Standish, E., \& Williams, J. G. (n. d.). Orbital Ephemerides of the Sun, Moon and Planets (p. 16).

Nelson, R. A. (2011). Relativistic Time Transfer in the Vicinity of the Earth and in the Solar System. Metrologia, 48, S171. https://doi.org/10.1088/0026-1394/48/4/S07

Ostro, S. J. (1993). Planetary Radar Astronomy. Reviews of Modern Physics, $65,1235$. https://doi.org/10.1103/RevModPhys.65.1235

Petit, G., \& Wolf, P. (2005). Relativistic Theory for Time Comparisons: A Review. Metrologia, 42 , S138. https://doi.org/10.1088/0026-1394/42/3/S14

Post, E. J. (1967). Sagnac Effect. Reviews of Modern Physics, 39, 475. https://doi.org/10.1103/RevModPhys.39.475

Rindler, W. (2006). Relativity Special, General and Cosmological (2nd ed.). Oxford University Press, New York.

Sagnac, G. (1979). The Luminiferous Ether Demonstrated by the Effect of the Relative Motion of the Ether in an Interferometer in Uniform Rotation. C. R. Acad. Sci. Paris 157, 708, 1913, translated by R. Hazelett in the Einstein Myth and the Ives Papers ed., R. hazelett and D. Tuner, Devin-Adair, Connecticut.

Saito, Y. (2005). A Discussion of Roemer's Discovery Concerning the Speed of Light. AAPPS Bulletin, 9, 15.

Sardin, G. (2004). Testing Lorentz symmetry of special relativity by means of the Virgo or Ligo set-up, through the differential measure of the two orthogonal beams time-of-flight.

Sato, M. (2010). The Velocity of Electromagnetic Wave is Observed Differently Depending on the Observer's Velocity. Physics Essays, 23, 405. https://doi.org/10.4006/1.3452472

Schaffner, K. F. (1972). Nineteenth-Century Aether Theories. Pergamon Press, Oxford.

Selleri, F. (1997). Noninvariant One-way Speed of Light and Locally Equivalent Reference Frames. Foundation of Physics Letters, 10, 73. https://doi.org/10.1007/BF02764121

Selleri, F. (2004). Recovering the Lorentz Ether. Apeiron, 11, 246. 
Selleri, F. (2010). Sagnac Effect: End of the Mystery. In G. Rizzi \& M. L. Ruggiero (Eds.), Relativity in Rotating Frames (pp. 55-78). Kluwer Academic Publishers, London.

Selleri, F. (2011). La Relativita Debole. Edizioni Melquiades, Milano.

Shankland, R. S., Mc Cuskey, S. W., Leone, F. C., \& Kuerti, G. (1955). New analysis of the interferometer observations of Dayton C. Miller. Review of Modern Physics, 27, 167. https://doi.org/10.1103/RevModPhys.27.167

Stachel, J. (2005). 1905 and all that. Nature, 433, 215. https://doi.org/10.1038/433215a

$\mathrm{Su}$, C.-C. (2001). A Local-Ether Model of Propagation of Electromagnetic Wave. The European Physical Journal C, 21, 701. https://doi.org/10.1007/s100520100759

Wallace, B. G. (1969). Radar Testing of the Relative Velocity of Light in Space. Spectroscopy Letters, $2,361$. https://doi.org/10.1080/00387016908050941

Wang, R. (2000). Successful GPS Operations Contradict the Two Principles of Special Relativity and Imply a New Way for Inertial Navigation-Measuring Speed Directly. Proceedings of the IAN World Congress in Association with the U.S. ION Annual Meeting, 26-28 June 2000, San Diego, CA.

Whittaker, E. (1973). A History of the Theories of Aether and Electricity: The Classical Theories. Humanities Press, New York.

Xu, G. (2007). GPS Theory, Algorithms and Applications (2nd ed.). Springer-Verlag, Berlin.

Zhang, Y. Z. (1997). Special Relativity and its Experimental Foundations. World Scientific, Singapore. https://doi.org/10.1142/3180

\section{Copyrights}

Copyright for this article is retained by the author(s), with first publication rights granted to the journal.

This is an open-access article distributed under the terms and conditions of the Creative Commons Attribution license (http://creativecommons.org/licenses/by/4.0/). 